\title{
Binding of autoantibodies and apoptotic response in erythroid cells in the mouse model of autoimmune hemolytic anemia
}

\begin{abstract}
C57BL/6 mice were given repeated weekly injections of rat erythrocytes to induce autoimmune hemolytic anemia (AIHA), characterized by decreased erythrocyte count and hemoglobin content, presence of membrane bound autoantibodies on circulating erythrocytes and a decline in the bone marrow erythropoietic activity. Erythroid cells in the bone marrow and spleen of AIHA mice were examined for the presence of membrane bound autoantibodies using a fluorochrome conjugated anti-mouse $\operatorname{IgG}$ antibody. Apoptotic activity was also assessed flow cytometrically using Annexin V and 7AAD. Results showed that there is significant binding of autoantibodies to both spleen and bone marrow, but the apoptosis is evident only in bone marrow, that too in later stages of differentiation.
\end{abstract}

Keywords: autoantibody, erythropoiesis, apoptosis, hemoglobin, intraperitoneal injection
Volume 5 Issue 2 - 2017

\author{
Sreoshi Chatterjee,' Rajiv K Saxena ${ }^{2}$ \\ 'Department of Life Sciences, Jawaharlal Nehru University, India \\ ${ }^{2}$ Department of Life Sciences and Biotechnology, South Asian \\ University, India
}

\begin{abstract}
Correspondence: Rajiv K Saxena, Faculty of Life Sciences and Biotechnology, South Asian University, New Delhi, India,
\end{abstract} Email rajivksaxena@hotmail.com

Received: December 08, 2016 | Published: September 05, 2017
Abbreviations: AIHA, autoimmune hemolytic anemia; DIB, double in vivo biotinylation; BM, bone marrow; PS, phosphatidylserine; ROS, reactive oxygen species; MFI, mean fluorescence intensity; SEM, standard error of the mean; SOP, standard operating procedures; SAv-APC, streptavidin-allophycocyanin; NHS, N-hydroxysuccinimide; 7AAD, 7-aminoactinomycin D; FITC, fluorescein isothiocyanate; DMF, dimethylformamide; FBS, fetal bovine serum; EDTA, ethylene diamine tetra acetic acid; DIB, double In vivo biotinylation; $\mathrm{BXN}$, biotin-X-NHS

\section{Introduction}

Autoimmune hemolytic anemia (AIHA), characterized by the presence of self-reactive autoantibodies against erythrocytes, ${ }^{1-3}$ can be induced in C57BL/6 mice by repeated intraperitoneal injection of rat erythrocytes ${ }^{4-6}$ following the Playfair and Clarke Model. ${ }^{7}$ Pathogenesis of AIHA involves two underlying mechanisms, viz, erythrophagocytosis of autoantibody (IgG) coated erythrocytes by macrophages in the reticuloendothelial system in liver and spleen, ${ }^{8-9}$ and complement mediated lysis of erythrocytes with membranebound IgM autoantibodies. ${ }^{10}$ AIHA has been extensively studied for the pathogenesis, clinical features and prognosis of the disease. ${ }^{3,11}$ However the modulation of erythropoietic homeostasis and the turnover pattern of circulating erythrocytes are poorly understood. In a recent work from our group, Chatterjee et al. ${ }^{6}$ have studied all the different stages of erythroid system, from early proerythroblasts to reticulocytes, to the different age groups of circulating erythrocytes in AIHA mice ${ }^{6}$ and have unraveled a link between the autoantibody binding and the decline in erythroid cells. The overall decline in erythropoietic activity and erythrocyte count as evidenced in this study was characterized by a higher susceptibility of the more differentiated erythroblasts $\mathrm{C}$ and the youngest erythrocytes in circulation. ${ }^{6}$ However the apoptotic activity, which plays a crucial role in the destruction of cells, was not studied in these erythroid cells. In the present study we set onto study a correlation, if any, between the membrane-bound autoantibody and the apoptotic activity in the erythroid cells.
Although erythrocytes lack nucleus, a process similar to apoptosis, termed 'eryptosis' has been identified in erythrocytes. ${ }^{12,13}$ Circulating erythrocytes that are damaged to an extent that would compromise their integrity, even before reaching senescence, undergo a mechanism of a regulated and coordinated suicidal death, termed 'eryptosis ${ }^{13,14}$, Eryptosis is very similar to apoptosis, the programmed cell death in nucleated cells, ${ }^{15}$ which involves cell shrinkage, membrane blebbing, and plasma membrane scrambling leading to phosphatidylserine (PS) externalization. ${ }^{14}$ However since erythrocytes lack a nucleus and organelles, eryptosis also lacks several hallmarks of apoptosis, like mitochondrial depolarization and nuclear condensation. Just like apoptotic cells, PS-exposing eryptotic cells are recognized and rapidly cleared from the circulation through erythrophagocytosis by macrophages. ${ }^{16,17}$ Disturbance of erythrocyte membrane lipids asymmetry and externalization of PS in mature erythrocytes is often triggered oxidative stress. ${ }^{18}$ Oxidative stress and high levels of reactive oxygen species (ROS) has been linked to autoimmunity in a variety of diseases ${ }^{19-21}$ and has also been shown as a critical factor in the rat erythrocyte administered mouse model of AIHA. ${ }^{6}$ PS externalization was therefore studied as a marker for both apoptotic and eryptotic activities in erythroid cells in AIHA mice.

\section{Materials and methods}

\section{Animals}

Inbred C57BL/6 male mice ( 8 -12weeks old, $20-25 \mathrm{~g}$ body weight) and female Wistar rats (2months old, 250-300g body weight) were used throughout this study. Animals were bred and maintained in microbe free environment in the animal house facility at Jawaharlal Nehru University (JNU), New Delhi or obtained from the National Institute of Nutrition (NIN), Hyderabad. The animals were housed in positive-pressure air conditioned units $\left(25^{\circ} \mathrm{C}, 50 \%\right.$ relative humidity) and kept on a $12 \mathrm{~h}$ light/dark cycle. Water and mouse chow were provided ad libitum. All the experimental protocols were conducted strictly in compliance with the Standard Operating Procedures (SOP) 
for Institutional Animal Ethics Committee (IAEC) of the Committee for the Purpose of Control and Supervision on Experiments on Animals (CPCSEA), Ministry of Environment, Forest and Climate Change, Govt. of India. The study was duly approved by Institutional Animal Ethics Committee of Jawaharlal Nehru University (IAEC Approved Project Code: 35/2012). All mice were randomly assigned to experimental groups. Experiments were designed so as to use the minimum number of mice.

\section{Reagents and other supplies}

Biotin-X-NHS (N-hydroxysuccinimide ester of biotin) was obtained from Sigma Aldrich (St. Louis, MO, USA). StreptavidinAllophycocyanin (SAv-APC), rat anti-mouse Ter-119-APC, rat anti-mouse CD71-Fluorescein isothiocyanate (FITC) monoclonal antibodies, anti-mouse CD16/CD32 purified and Annexin V-PE recombinant proteins were purchased from BD Biosciences (San Diego, CA, USA) or from Affymetrix eBioscience (San Diego, CA, USA). Goat F(ab')2 anti-mouse IgG-PE polyclonal antibody, rat IgG1 $\kappa$ FITC and rat IgG2bк-APC isotype controls, and 7-Aminoactinomycin D (7AAD) were procured from Affymetrix eBioscience (San Diego, CA, USA). Fetal bovine serum (FBS) was obtained from Hyclone (South Logan, UT, USA). RPMI was obtained from Sigma-Aldrich (St. Louis, MO, USA). HEPES, Dimethylformamide (DMF), Ethylene diamine tetra acetic acid (EDTA) and other analytical reagents were from Sigma-Aldrich (India). All other chemicals were purchased locally and were of analytical grade.

\section{Induction of autoimmune hemolytic anemia in mice}

Experimental autoimmune hemolytic anemia (AIHA) was induced in mice following the Playfair and Clarke model of repeated injections with rat erythrocytes..$^{47}$ Rat RBCs derived from tail vein were washed 3 times in phosphate-buffered saline (PBS, pH 7.4) and adjusted to a concentration of $1 \times 10^{9} \mathrm{cell} / \mathrm{s} / \mathrm{ml}$. Mice were given weekly injections of $2 \times 10^{8}$ rat RBCs intraperitoneally for 5weeks. Membrane-bound autoantibodies on erythrocytes were detected flow cytometrically by staining with $\mathrm{F}\left(\mathrm{ab}\right.$ ')2-anti-mouse IgG-PE. ${ }^{22-23}$

\section{Double in vivo biotinylation (DIB) of erythrocytes}

Double in vivo biotinylation (DIB) of erythrocytes was done as described previously. ${ }^{6,24-32}$ The DIB technique involves two steps of intravenous (i.v.) administration of biotin-X-NHS ester (BXN), through the tail vein of mice. In the first step of high intensity in vivo biotinylation, 3 daily i.v. injections of biotin ( $1 \mathrm{mg} \mathrm{BXN}$ dissolved in $20 \mu 1$ of DMF and $250 \mu 1$ of PBS) were given, followed after 30 days, by a low intensity biotinylation with a single lower dose $(0.6 \mathrm{mg} \mathrm{BXN}$ in $12 \mu 1 \mathrm{DMF}$ and $250 \mu 1 \mathrm{PBS}$ ). This low intensity biotinylation labels the fresh erythrocytes that were released in circulation in the 30-day period between the two biotinylation steps. At any time point after the second biotinylation, biotin intensity on circulating erythrocytes could be analyzed by flow cytometry using streptavidin coupled to any appropriate fluorochrome ${ }^{24,29}$ Biotin $^{\text {negative }}$ erythrocytes in circulation would represent the youngest erythrocytes released in blood after the second biotinylation step. Biotin ${ }^{\text {low }}$ erythrocytes would represent the cohort of erythrocytes released in circulation between the first and the second steps of biotinylation, and the biotin ${ }^{\text {high }}$ erythrocytes would represent the population of old residual erythrocytes that were present in blood during the first biotinylation. ${ }^{29}$ The DIB technique is summarized in Supplementary Figure 1.

\section{Erythroid differentiation in bone marrow and spleen}

For deriving bone marrow (BM) and spleen cells, mice were euthanized by $\mathrm{CO}_{2}$ asphyxiation before the organs were dissected out. BM cells were flushed out of femur and tibia using a 25 -gauze needle and re-suspended in RPMI medium with $10 \%$ FBS. Single cell suspensions of spleen cells were made by gently teasing the spleen in a small volume of PBS. Splenic and BM cells were strained through a fine sieve, pelleted by centrifugation, and re-suspended at desired concentration in RPMI with $10 \%$ FBS. For delineating erythroid precursors at different stages of differentiation, freshly prepared single cell suspensions from BM or spleen were first incubated with anti-CD16/32 monoclonal antibody (Fc block, $1 \mu \mathrm{g} / 10^{6}$ cells in $50 \mu 1$ of $\mathrm{PBS}+2 \% \mathrm{FBS}$ ) for $10 \mathrm{mins}$ followed by staining with anti-mouse CD71-FITC and anti-mouse Ter-119-APC for 20 mins at $4^{\circ} \mathrm{C} ., 32-34$

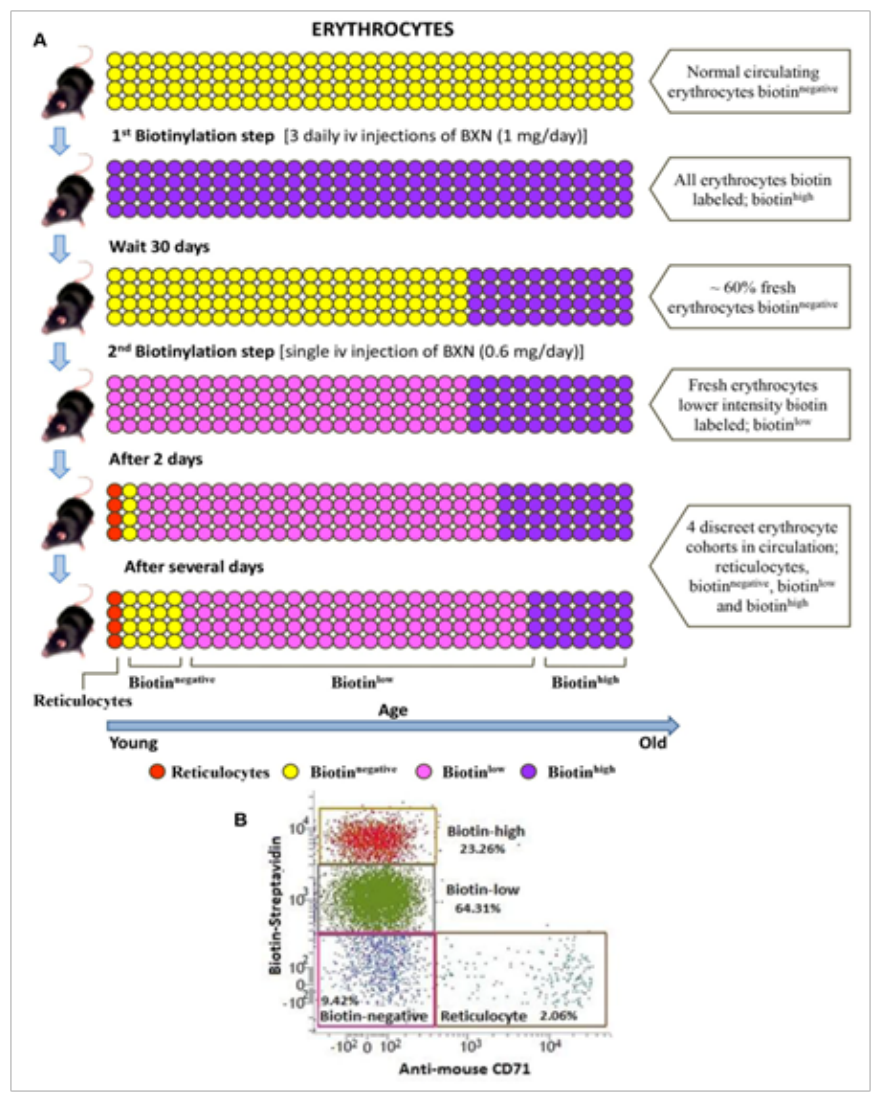

Supplementary Figure I Double in vivo biotinylation (DIB) for tracking age related changes on circulating erythrocytes.

C57BL/6 mice were administered three daily i.v. doses of Img BXN (I ${ }^{\mathrm{st}}$ biotinylation step). After a rest for 30days, a single additional dose of $0.6 \mathrm{mg}$ $B X N$ was administered ( $2^{\text {nd }}$ biotinylation step). Blood was collected at different time points and distribution of biotin label on erythrocytes was examined by staining the cells with streptavidin-APC followed by flow cytometry. Time schedule for the experiment is given in panel A. Principle of DIB protocol B is given in panel $B$, and Biotin label on circulating erythrocytes at different time points is given in panel $C$. Erythrocyte populations in boxes $X, Y$ and $Z$ represent biotin ${ }^{\text {high }}$, biotin ${ }^{\text {low }}$ and biotin ${ }^{\text {ngative }}$ populations respectively. Values in parentheses represent percentage of cells in different boxes.

\section{Determination of phosphatidylserine (PS) exposing erythroid cells}

Circulating erythrocytes and erythroid cells from BM and spleen that express Phosphatidylserine (PS) on their surface were estimated by incubating the cells with fluorochrome conjugated Annexin V recombinant protein along with 7AAD.$^{32}$ For the in vitro assay, mouse erythrocytes ( $2 \%$ suspension) were incubated with serum isolated 
from AIHA mice in the 96 well $\mathrm{U}$ bottom culture plates for $2 \mathrm{hrs}$ at $37^{\circ} \mathrm{C}$ and $5 \% \mathrm{CO}_{2}$. For $\mathrm{BM}$ and splenic assay single cell suspension of erythroid cells were prepared. These cells were washed and resuspended in Annexin V binding buffer (10mM Hepes, $\mathrm{pH} 7.4$, $140 \mathrm{mM} \mathrm{NaCl}, 2.5 \mathrm{mM} \mathrm{CaCl}_{2}$ ), followed by staining with 7AAD and Annexin V-PE for $20 \mathrm{mins}$ at $4^{\circ} \mathrm{C}$. After incubation, erythrocytes were washed and re-suspended in $\mathrm{HBS}$ with $2.5 \mathrm{mM} \mathrm{CaCl}_{2}$ and analyzed on flow cytometer immediately. Apoptotic/eryptotic cells were identified as $7 \mathrm{AAD}^{-}$Annexin $\mathrm{V}^{+}$cells.

\section{Flow cytometric analysis}

Mouse blood was collected in PBS containing 5mM EDTA and washed 3 times with ice cold saline containing HEPES buffer $(10 \mathrm{mM}$, $\mathrm{pH}-7.4)$ and $2 \%$ FBS. Biotin-labeled erythrocytes $\left(1 \times 10^{6}\right)$ were stained ex vivo with streptavidin-APC and anti-mouse CD71-FITC in dark for $30 \mathrm{mins}$ to identify the different age cohorts of erythrocytes, as described before. ${ }^{24,29}$ For enumerating erythroid cells at different stages of differentiation in BM and spleen the technique of double staining with anti-mouse CD71 and anti-mouse Ter119 was used, as described before. ${ }^{6,32-34}$ Briefly freshly prepared single cell suspensions $\left(1 \times 10^{6}\right)$ from BM or spleen were incubated with anti-mouse CD16/ CD32 antibody for $10 \mathrm{~min}$ followed by staining with anti-mouse CD71-FITC and anti-mouse Ter-119-APC for $20 \mathrm{~min}$ in dark at $4^{\circ} \mathrm{C}$.

To determine apoptotic marker PS externalization, erythroid cells $\left(1 \times 10^{6}\right)$ were co-stained with Annexin V-PE and 7AAD. To detect autoantibodies bound to erythroid cells, rat serum was used for blocking instead of mouse serum, and cells were stained with $\mathrm{F}(\mathrm{ab}$ ')2 anti-mouse IgG-PE. The cells were appropriately gated on the basis of streptavidin/CD71 staining or CD71/Ter119 staining, and the corresponding expression was assessed. All the stained cells were washed and analyzed immediately on a flow cytometer. For all the flow cytometric analysis 7AAD was used as viability dye and immunophenotyping was carried out on gated live 7AAD- cells. A minimum of 50,000 events were recorded. All the flow cytometric analyses were performed on a BD FACSVerse flow cytometer (Becton Dickinson, San Jose, CA, USA) using FACSuite software for acquisition and analysis.

\section{Statistical analysis}

Each experiment was repeated at least three times. Statistical analysis by Student's t-test and ANOVA was carried out using SigmaPlot software. Data are presented as means \pm SEM. A level of $p<0.05$ was accepted as statistically significant.

\section{Results and discussion}

\section{Binding of autoantibodies and phosphatidylserine externalization in circulating erythrocytes in AIHA mice}

C57BL/6 mice were immunized weekly with rat erythrocytes intraperitoneally for 5-6weeks to induce autoimmune hemolytic anemia (AIHA). Generation of anti-mouse erythrocyte autoantibody was estimated by flow cytometric analysis of erythrocytes stained with anti-mouse IgG/IgM-FITC polyclonal antibodies. The anti-mouse erythrocyte autoantibody level showed a sharp increase of $60 \%$ in the MFI of the FITC-conjugated secondary antibody, from $7.10 \pm 0.25$ in control to $11.46 \pm 0.74$ in mice after 5 immunizations. The proportion of erythrocytes with membrane-bound anti-mouse autoantibody also increased, almost 3 -fold from $3.5 \pm 1.11 \%$ to $10.3 \pm 1.86 \%$ (Figure 1 ).

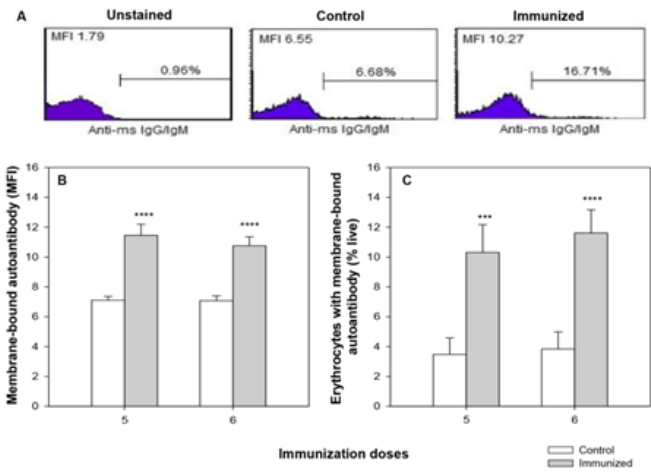

Figure I Generation of anti-mouse erythrocyte autoantibody in mice with induced AlHA.

C57BL/6 mice were given intraperitoneal injections of $2 \times 10^{8}$ rat erythrocytes weekly for 5-6weeks to induce autoimmune hemolytic anemia (AlHA). At intended time points mice were bled and erythrocytes $\left(I \times \mid 0^{6}\right)$ were stained with anti-mouse IgG/IgM-FITC polyclonal antibody to assess the presence of membrane-bound autoantibody in erythrocytes in control and immunized mice. Representative flow histograms showing anti-mouse lgG/lgM-FITC staining is given in panel $A$. Presence of membrane-bound autoantibody on circulating erythrocytes in terms of binding (MFI) and proportion of erythrocytes with membrane-bound autoantibody is given in panels $B$ and $C$ respectively. Each bar in the graph represents mean \pm SEM of observations from 10 mice. $* * * p<0.005$ and $* * * * p<0.001$ for comparison of the groups (Student t-test).

Erythrocytes fromAIHAmice were examined for phosphatidylserine (PS) externalization. However, Annexin V staining in erythrocytes from control and immunized mice revealed no significant change, probably because $\mathrm{PS}^{+}$erythrocytes are readily removed from circulation. Therefore to assess the extent of PS externalization on erythrocyte membrane after binding of autoantibodies, an indirect method was employed, where erythrocytes from a separate healthy mouse were incubated for $2 \mathrm{hrs}$ with serum from AIHA mice, followed by staining with Annexin V-PE and 7AAD. $\mathrm{PS}^{+}$erythrocytes were identified as Annexin $\mathrm{V}^{+} 7 \mathrm{AAD}^{-}$cells. Simultaneous staining with anti-mouse IgG-FITC would indicate the binding of autoantibodies to these erythrocytes. To examine the PS externalization in different age groups of erythrocytes, the donor mouse was administered double in vivo biotinylation (DIB, concept explained in Supplementary Figure 1) before the process. Briefly, in this DIB protocol, $, 624,29,32$ mice were given the two i.v. biotin injections at an interval of 30days, and the blood was collected after 10 days of second biotinylation, to get two distinct subgroups of very young biotin ${ }^{\text {negative }}(<10$ days old $)$ and very old biotin ${ }^{\text {high }}$ ( $>40$ days old) erythrocytes. After incubation with serum from AIHA mice, the erythrocytes were stained with streptavidin APC, CD71-FITC, Annexin V-PE and 7AAD. Different age groups of erythrocytes were gated and proportion of $\mathrm{PS}^{+}$cells were determined in each of these cohorts. Results in Table 1 revealed a marginal, yet significant increase in the proportion of $\mathrm{PS}^{+}$cells in the younger cohorts (biotin ${ }^{\text {negative }}$ and reticulocytes) of erythrocytes in mice with induced AIHA. The extent of PS externalization in the aged erythrocytes remained more or less unchanged. This pattern was similar to the extent of autoantibody binding in these cells.

\section{Binding of autoantibodies and phosphatidylserine externalization in erythroid cells in bone marrow and spleen of AIHA mice}

Bone marrow (BM) and spleen, the two prime erythropoietic sites 
in adult mice, were examined for the presence of membrane-bound autoantibody and apoptotic activity. Erythroid line of differentiation in bone marrow and spleen starts with the early progenitor proerythroblasts that are derived from the pluripotent stem cells. Proerythroblasts further differentiate in successive stages viz erythroblast A, B and C. ${ }^{35,36}$ Differential expression of Ter119 and CD71 molecules on erythroid cell surface in BM and spleen were used to delineate four distinct stages of erythroid differentiation: early pro-erythroblasts (Ter119 ${ }^{\text {med }}$ CD71 $1^{\text {high }}$ ), early basophilic erythroblasts (Ter119 $9^{\text {high }}$ Table I Phosphatidylserine (PS) externalization in erythrocytes
CD71 $1^{\text {high }} \mathrm{FSC}^{\text {high }}$, erythroblast A), late basophilic, polychromatic and orthochromatic erythroblasts (Ter119 $9^{\text {high }} \mathrm{CD} 71^{\text {med }}$ FSC low , erythroblast B) and orthochromatic erythroblasts with mature erythrocytes (Ter119 ${ }^{\text {high }} \mathrm{CD} 71^{\text {low }} \mathrm{FSC}^{\text {low }}$, erythroblast C), as described elsewhere ${ }^{6,32-34}$ (Supplementary Figure 2). Total erythroid population in BM and spleen was delineated and enumerated by setting an inverted L-shaped gate in the flow histogram that includes all the above mentioned four stages of erythroid differentiation (Supplementary Figure 2). ${ }^{33,34}$

\begin{tabular}{lllll}
\hline \multirow{2}{*}{ Erythrocyte age cohorts } & \multicolumn{2}{l}{ PS+Erythrocytes $(\%)$} & \multicolumn{2}{l}{ Autoantibody (MFI) } \\
\cline { 2 - 5 } & Control sera & Immunized sera & Control sera & Immunized sera \\
\hline Total erythrocytes & $1.06 \pm 0.31$ & $1.18 \pm 0.08$ & $24.7 \pm 1.20$ & $32.7 \pm 2.12^{*}$ \\
Biotin $^{\text {high }}\left(S A v^{\text {high }} C D 7 I^{\text {low }}\right)$ & $2.86 \pm 0.95$ & $2.73 \pm 0.19$ & $29.3 \pm 1.45$ & $33.7 \pm 1.41$ \\
Biotin $^{\text {low }}\left(S A v^{\text {med }} C D 7 I^{\text {low }}\right)$ & $0.88 \pm 0.45$ & $0.96 \pm 0.11$ & $22.3 \pm 0.88$ & $30.2 \pm 2.08^{*}$ \\
Biotin $^{\text {negative }}\left(\left.S A v^{\text {low }} C D 7\right|^{\text {low }}\right)$ & $0.27 \pm 0.02$ & $0.45 \pm 0.04^{*}$ & $21.7 \pm 1.45$ & $31.7 \pm 2.49^{*}$ \\
Reticulocyte $\left(\left.S A v^{\text {low }} C D 7\right|^{\text {high }}\right)$ & $1.32 \pm 0.19$ & $2.35 \pm 0.17^{* *}$ & $67.3 \pm 2.40$ & $98.3 \pm 4.58^{* * *}$ \\
\hline
\end{tabular}

Mice were given weekly i.p. injections of rat erythrocytes for 5 weeks to induce AlHA, blood samples were collected thereafter, and serum isolated. A separate group of mice were given biotin injections as per schedule in Supplementary figure I, IOdays after the $2^{\text {nd }}$ biotinylation, erythrocytes were collected and incubated in vitro with the sera from control and AlHA mice. Erythrocytes were then washed and stained with streptavidin-APC, anti-mouse CD7I-FITC and Annexin V-PE. Erythrocytes of different age groups were gated and the PS externalization and presence of membrane-bound autoantibody was assessed in each of these groups. Each value represents mean \pm SEM of observations. $n=3$ control and 7 immunized sera. $* p<0.05$, $* * p<0.01$ and $* * * p<0.005$ for comparison of the groups (Student t-test).

A

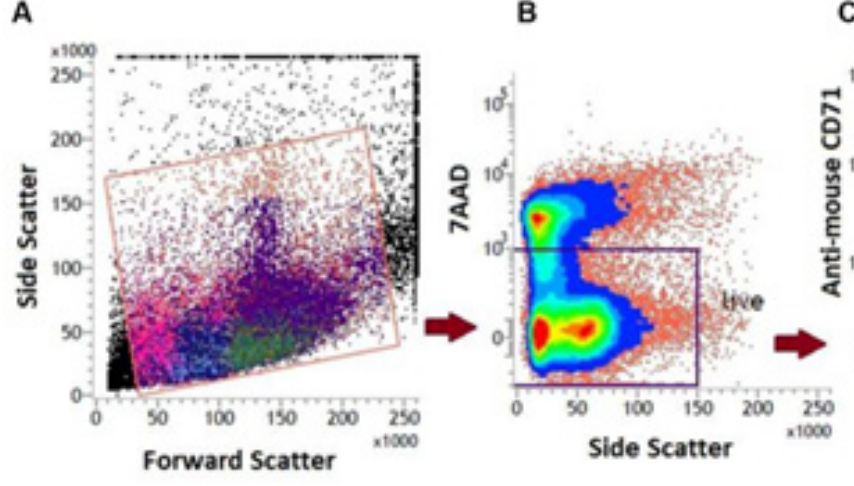

C

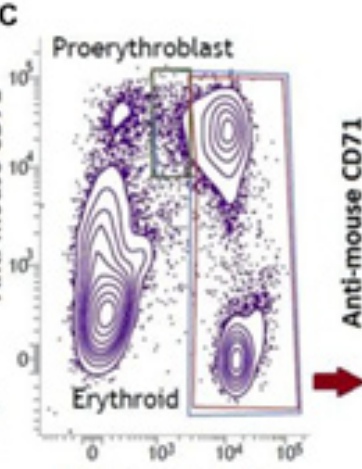

D

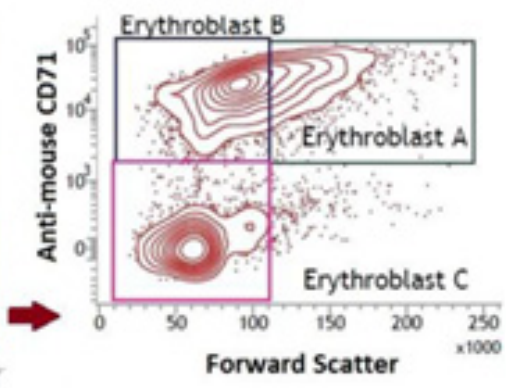

Anti-mouse Ter119

Supplementary Figure 2 Erythropoietic activity in the bone marrow (BM) and spleen in mice with induced AlHA.

Mice were given intraperitoneal injections of $2 \times 10^{8}$ rat erythrocytes weekly for $5-6$ weeks to induce AlHA. Mice were sacrificed 3 days after $5^{\text {th }}$ and $6^{\text {th }}$ immunizations and their BM and spleen cells were harvested. Cells isolated were stained with anti-mouse CD7I-PE, anti-mouse Ter I I9-APC and 7AAD, and the proportions of erythroid cells were determined. Four distinct stages of erythroid differentiation could be identified within an inverted 'L' shaped gate in the flow diagram. These include early proerythroblasts (Ter I I $9{ }^{\mathrm{med}} \mathrm{CD} 7 \mathrm{I}^{\text {high }}$ ), early basophilic erythroblasts or erythroblasts A (Ter I $9^{\text {high }} \mathrm{CD} 7 \mathrm{I}^{\text {high }} \mathrm{FSC}$ high), late basophilic polychromatic and orthochromatic erythroblasts or erythroblasts B (Ter I I $9^{\text {high }}$ CD7I ${ }^{\text {medFSClow }}$ ), and orthochromatic erythroblasts with mature erythrocytes or erythroblasts $\mathrm{C}$ (TerII $\left.9^{\text {high }} \mathrm{CD} 7 \mathrm{I}^{\text {low }} \mathrm{FSC}^{\text {low }}\right)$.

In our previous study, ${ }^{6}$ examination of erythroid cells for membrane-bound autoantibody revealed a significant increase in the autoantibody binding in both BM and spleen in the total erythroid population (Figure 2) as well as the different stages of differentiation (more significant in erythroblasts B and C). As such presence of membrane-bound autoantibodies on erythroid cells, particularly in $\mathrm{BM}$, has been regarded as a critical factor for the decline in overall erythropoietic activity in the AIHA mice. This observation was in line with several earlier studies on SLE (systemic lupus erythematosus), where presence of inhibitory autoantibody on BM progenitor cells had been linked to the development of hypoplasia, and even pure red cell aplasia. ${ }^{37,38}$ In the present study erythroid cells in BM and spleen were examined for their apoptotic response. Annexin-V staining showed a marked increase in the proportion of apoptotic cells (Annexin- $\mathrm{V}^{+}$ $7 \mathrm{AAD}^{-}$) in $\mathrm{BM}$ erythroid cells (from $4.20 \pm 0.27 \%$ in control to $5.16 \pm 0.40 \%$ in AIHA mice; Figure 3 ). This increase was also evident in the erythroblasts B and C subpopulations in BM, which showed a $30 \%$ increase in the proportion of apoptotic cells (Figure 4). The apoptotic activity in BM therefore followed the same pattern as observed in case of membrane-bound autoantibody. The splenic erythroid cells however showed no change in apoptotic response (Figure 3) \& (Figure 4). 


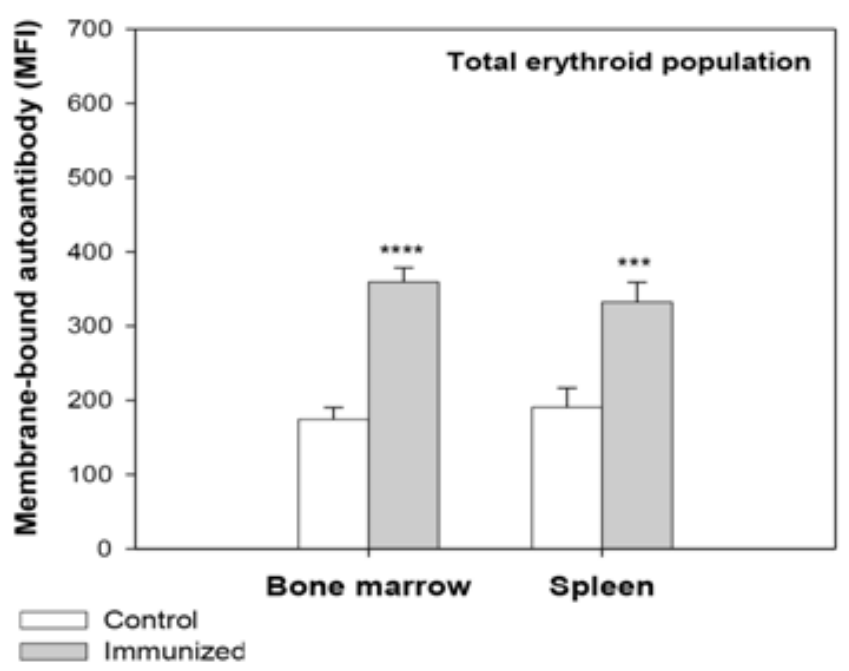

Figure 2 Presence of membrane-bound anti-mouse autoantibody on erythroid cells of bone marrow (BM) and spleen.

Mice were given intraperitoneal injections of $2 \times 10^{8}$ rat erythrocytes weekly for 5-6weeks to induce AlHA. Mice were sacrificed and their BM and spleen cells harvested. Cells isolated were stained with anti-mouse CD7I-PE, anti-mouse Terl I9-APC and 7AAD, to determine the proportion of live erythroid cells. The erythroid cells were co-stained with $F(a b$ ') 2 anti-mouse IgG-PE to detect the presence of autoantibodies. Presence of membrane-bound autoantibody in the total erythroid populations of BM and spleen after 5 immunizations is shown above (ANOVA test for both BM and spleen, $p<0.00 \mathrm{I}$ ). Each bar on the graph represents mean \pm SEM of observations. $n=4$ control and 6 AlHA mice. $* * * p<0.005$ and $* * * * p<0.00$ I for comparison of groups (Student t-test).

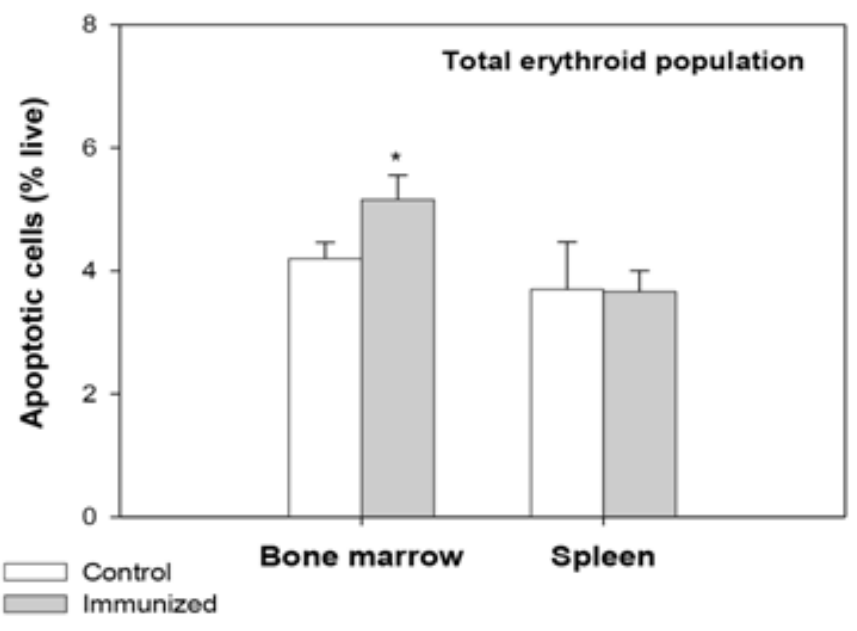

Figure 3 Apoptotic response in erythroid cells of BM and spleen in mice with induced AlHA.

Mice were given intraperitoneal injections of $2 \times 10^{8}$ rat erythrocytes weekly for 5-6weeks to induce AIHA. Mice were sacrificed and their BM and spleen cells were harvested. Cells isolated were stained with anti-mouse CD7IFITC, anti-mouse TerII9-APC and 7AAD, to determine the proportion of live erythroid cells. Erythroid cells were incubated with Annexin V-PE and the apoptotic cells were identified as Annexin V+7AAD- cells. Proportion of apoptotic cells in the whole erythroid population in BM and spleen is given above. Each bar on the graph represents mean \pm SEM of observations. $n=4$ control and 6 AlHA mice. ${ }^{*} p<0.05$ for comparison of groups (Student t-test).

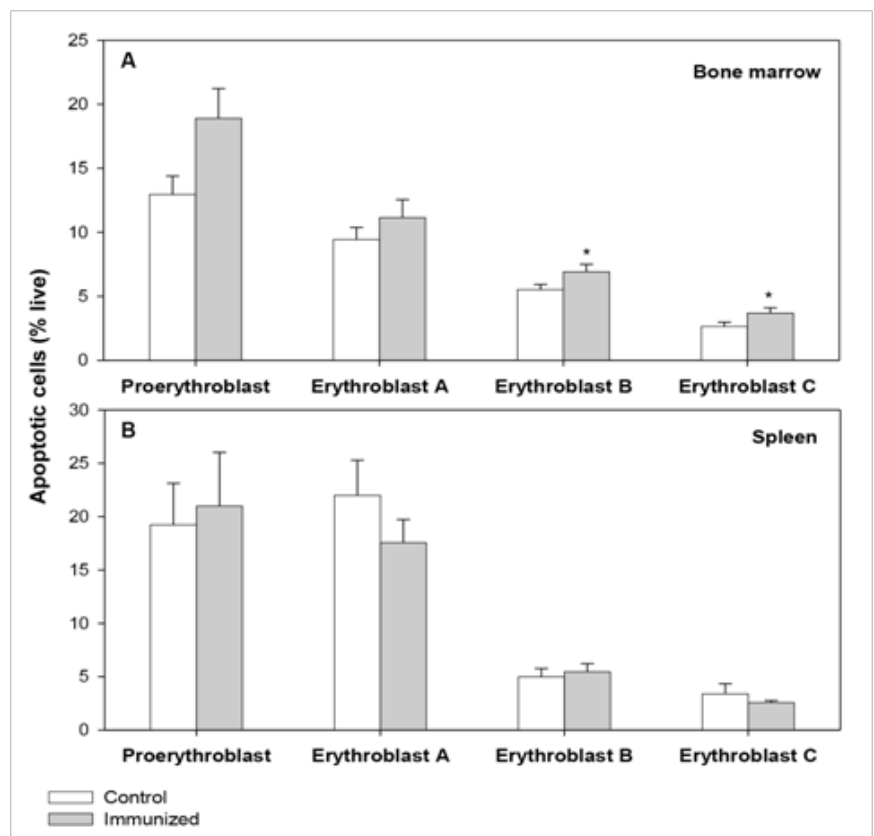

Figure 4 Apoptotic response in erythroid cells of BM and spleen at different stages of maturation.

Mice were given intraperitoneal injections of $2 \times 10^{8}$ rat erythrocytes weekly for 5-6weeks to induce AlHA. Mice were sacrificed and their BM and spleen cells were harvested. Cells isolated were stained with anti-mouse CD7I-FITC, antimouse Ter I 19-APC and 7AAD, to determine the proportion of live erythroid cells. Erythroid cells were incubated with Annexin V-PE and the apoptotic cells were identified as Annexin $\mathrm{V}^{+} 7 \mathrm{AAD}^{-}$cells. Apoptotic cells in erythroid populations at different stages of maturation in BM and spleen are shown in panels $A$ and $B$ respectively. Each bar on the graph represents mean \pm SEM of observations. $n=4$ control and 6 AlHA mice. $* p<0.05$ for comparison of groups (Student t-test).

\section{Conclusion}

Autoimmune anemia is characterized by the presence of antierythrocyte autoantibodies, ${ }^{5}$ which is evident from the presence of membrane-bound autoantibodies on circulating erythrocytes. These autoantibodies seem to be directed not only against the mature erythrocytes, but also the erythroid precursors at every stage of differentiation. ${ }^{6}$ Since binding of autoantibody on the erythroid membrane could cause a membrane perturbation, it might also trigger an apoptotic response in these erythroid cells. Additionally autoantibody binding has been shown to be linked with enhanced oxidative stress, ${ }^{6,19-21}$ and oxidative stress on the other hand, is known to be a critical factor triggering apoptosis. ${ }^{18}$

In this study apoptotic activity in erythroid progenitors and eryptotic activity in mature non-nucleated erythrocytes were examined as a consequence of autoimmune response in AIHA mice. Results showed increased apoptotic/eryptotic activities in erythroid cells with the highest titer of membrane-bound autoantibodies. Although membrane-bound autoantibodies could be evidenced in each and every stage of erythroid system, this selective activation of apoptosis/eryptosis in erythroid cells could indicate a critical density of autoantibodies that is necessary to elicit the apoptotic response. As such, only the cells with highest deposition of autoantibodies on their membrane show significant apoptotic activity, which might be a causative factor for their selective destruction. Splenic erythroid 
cells in AIHA mice however, did not show any apoptotic activity in spite of very high titer of autoantibodies on their membrane. Similar trend was also observed for the intracellular ROS generation in these cells. ${ }^{6}$ The splenic population might harbor protective mechanisms to counteract the perils of autoantibody binding that might also be linked to the activation of stress response in spleen erythroid cells in conditions of BM hypoplasia/aplasia and acute anemia. This requires further studies.

\section{Acknowledgements}

The work was funded by two research grants from the Department of Science and Technology to RKS. SC received Senior Research Fellowship from Council of Scientific and Industrial Research (CSIR), New Delhi.

\section{Conflict of interest}

The author declares no conflict of interest.

\section{References}

1. Sokol RJ, Hewitt S. Autoimmune hemolysis: a critical review. Crit Rev Oncol Hematol. 1985;4(2):125-154.

2. Hashimoto C. Autoimmune hemolytic anemia. Clin Rev Allergy Immunol. 1998;16(3):285-295.

3. Gehrs BC, Friedberg RC. Autoimmune hemolytic anemia. Am J Hematol. 2002;69(4):258-271.

4. Cox KO, Keast D. Autoimmune haemolytic anaemia induced in mice immunized with rat erythrocytes. Clin Exp Immunol. 1974;17(2):319327.

5. Naysmith JD, Ortega-Pierres MG, Elson CJ. Rat erythrocyte induced anti-erythrocyte autoantibody production and control in normal mice. Immunol Rev. 1981;55:55-87.

6. Chatterjee S, Bhardwaj N, Saxena RK. Identification of stages of erythroid differentiation in bone marrow and erythrocyte subpopulations in blood circulation that are preferentially lost in autoimmune hemolytic anemia in mouse. PLoS One. 2016;11(11):e0166878.

7. Playfair JH, Marshall-Clarke S. Induction of red cell autoantibodies in normal mice. Nat New Biol. 1973;243(128):213-214.

8. Engelfriet CP, Overbeeke MA, von dem Borne AE. Autoimmune hemolytic anemia. Semin Hematol. 1992;29(1):3-12.

9. Izui S, Berney T, Shibata $\mathrm{T}$, et al. Molecular and cellular basis for pathogenicity of autoantibodies. Tohoku J Exp Med. 1994;173(1):15-30.

10. Jefferies LC. Transfusion therapy in autoimmune hemolytic anemia. Hematol Oncol Clin North Am. 1994;8(6):1087-1104.

11. Semple JW, Freedman J. Autoimmune pathogenesis and autoimmune hemolytic anemia. Semin Hematol. 2005;42(3):122-130.

12. Kuypers FA, De Jong K. The role of phosphatidylserine in recognition and removal of erythrocytes. Cell Mol Biol (Noisy-le-grand). 2004;50(2):147-158.

13. Lang KS, Lang PA, Bauer C, et al. Mechanisms of suicidal erythrocyte death. Cell Physiol Biochem. 2005;15(5):195-202.

14. Lang F, Gulbins E, Lang PA, Zappulla D, et al. Ceramide in suicidal death of erythrocytes. Cell Physiol Biochem 26(1): 21-28.

15. Bratosin D, Estaquier J, Petit F, Arnoult D, Quatannens B, et al Programmed cell death in mature erythrocytes: a model for investigating death effector pathways operating in the absence of mitochondria. Cell Death Differ. 2001;8(12):1143-1156.
16. Schroit AJ, Madsen JW, Tanaka Y. In vivo recognition and clearance of red blood cells containing phosphatidylserine in their plasma membranes. J Biol Chem. 1985;260(8):5131-5138.

17. McEvoy L, Williamson P, Schlegel RA. Membrane phospholipid asymmetry as a determinant of erythrocyte recognition by macrophages. Proc Natl Acad Sci U S A. 1986;83(10):3311-3315.

18. Föller M, Huber SM, Lang F. Erythrocyte programmed cell death. IUBMB Life. 2008;60(10):661-668.

19. Kurien BJ, Hensley K, Bachmann M, et al. Oxidatively modified autoantigens in autoimmune diseases. Free Radical Biology \& Medicine. 2006;41:549-556.

20. Iuchi Y, Kibe N, Tsunoda S, et al. Implication of oxidative stress as a cause of autoimmune hemolytic anemia in NZB mice. Free Radic Biol Med. 2010;48(7):935-944.

21. Fujii J, Kurahashi T, Konno T, et al. Oxidative stress as a potential causal factor for autoimmune hemolytic anemia and systemic lupus erythematosus. World J Nephrol. 2015;4(2):213-222.

22. Arndt PA, Garratty G. Flow cytometric analysis in red blood cell immunology. Transfus Med Hemother. 2004;31:163-174.

23. Thedsawad A, Taka O, Wanachiwanawin W. Development of flow cytometry for detection and quantitation of red cell bound immunoglobulin $\mathrm{G}$ in autoimmune hemolytic anemia with negative direct Coombs test. Asian Pac J Allergy Immunol. 2011;29(4):364-367.

24. Khandelwal S, Saxena RK. Assessment of survival of aging erythrocyte in circulation and attendant changes in size and CD147 expression by a novel two step biotinylation method. Exp Gerontol. 20006;41(9):855861.

25. Khandelwal S, van Rooijen N, Saxena RK. Reduced expression of CD47 during murine red blood cell (RBC) senescence and its role in $\mathrm{RBC}$ clearance from the circulation. Transfusion. 2007;47(9):1725-1732.

26. Khandelwal S, Saxena RK. A role of phosphatidylserine externalization in clearance of erythrocytes exposed to stress but not in eliminating aging populations of erythrocyte in mice. Exp Gerontol. 2008;43(8):764-770.

27. Saxena RK, Khandelwal S. Aging and destruction of blood erythrocytes in mice. Current science. 2009;97(4):500-507.

28. Sachar S, Saxena RK. Cytotoxic effect of poly-dispersed single walled carbon nanotubes on erythrocytes in vitro and in vivo. PLoS One. 2011;6(7):e22032.

29. Saxena RK, Bhardwaj N, Sachar S, et al. A Double in vivo biotinylation (DIB) technique for objective assessment of aging and clearance of mouse erythrocytes in blood circulation. Transfus Med Hemother. 2012;39(5):335-341.

30. Bhardwaj N, Saxena RK. Elimination of young erythrocytes from blood circulation and altered erythropoietic patterns during paraquat induced anemic phase in mice. PLoS One. 2014;9(6):e99364.

31. Bhardwaj N, Saxena RK. Selective loss of younger erythrocytes from blood circulation and changes in erythropoietic patterns in bone marrow and spleen in mouse anemia induced by poly-dispersed single wall carbon nanotubes. Nanotoxicology. 2015;9(8):1032-1040.

32. Chatterjee S, Saxena RK. Preferential elimination of older erythrocytes in circulation and depressed bone marrow erythropoietic activity to cadmium induced anemia in mice. PLoS One. 2015;10(7):e0132697.

33. Liu Y, Pop R, Sadegh C, et al. Suppression of Fas-FasL coexpression by erythropoietin mediates erythroblast expansion during the erythropoietic stress response in vivo. Blood. 2006;108(1):123-133.

34. Kalfa TA, Pushkaran S, Zhang X, et al. Rac1 and Rac2 GTPases are necessary for early erythropoietic expansion in the bone marrow but not in the spleen. Haematologica. 2010;95(1):27-35. 
35. Hattangadi SM, Wong P, Zhang L, et al. From stem cell to red cell: regulation of erythropoiesis at multiple levels by multiple proteins, RNAs, and chromatin modifications. Blood. 2011;118(24):6258-6268.

36. Dzierzak E, Philipsen S. Erythropoiesis: Development and Differentiation. Cold Spring Harb Perspect Med. 2013;3(4):a011601.
37. Roffe C, Cahill MR, Samanta A, et al. Aplastic anaemia in systemic lupus erythematosus: a cellular immune mechanism? Br J Rheumatol. 1991;30(4):301-304.

38. Kiely PD, McGuckin CP, Collins DA, et al. Erythrocyte aplasia and systemic lupus erythematosus. Lupus. 1995;4(5):407-411. 